\title{
On the Determination of Star and Convex Bodies by Section Functions*
}

\author{
R. J. Gardner, ${ }^{1}$ A. Soranzo, ${ }^{2}$ and A. Volčičํㄹ \\ ${ }^{1}$ Department of Mathematics, Western Washington University, \\ Bellingham, WA 98225-9063, USA \\ gardner@baker.math.wwu.edu \\ ${ }^{2}$ Dipartimento di Scienze Matematiche, Universitá degli Studi di Trieste, \\ 34100 Trieste, Italy \\ \{soranzo,volcic\}@univ.trieste.it
}

\begin{abstract}
The $i$ th section function of a star body in $\mathbb{E}^{n}$ gives the $i$-dimensional volumes of its sections by $i$-dimensional subspaces. It is shown that no star body is determined among all star bodies, up to reflection in the origin, by any of its $i$ th section functions. Moreover, the set of star bodies that are determined among all star bodies, up to reflection in the origin, by their $i$ th section functions for all $i$, is a nowhere dense set. The determination of convex bodies in this sense is also studied. The results complement and contrast with recent results on the determination of convex bodies by $i$ th projection functions. The paper continues the development of the dual Brunn-Minkowski theory initiated by Lutwak.
\end{abstract}

\section{Introduction}

An important concern in geometric tomography is the question of whether an object in a given class can be distinguished from others in the class by various measurements of its projections on planes or sections by planes. If this is the case, we say that the object is determined in the class by the data. For projections, the natural class of objects to consider is the class $\mathcal{K}^{n}$ of convex bodies in $\mathbb{E}^{n}$. One natural type of data is the $i$ th projection function of some $K \in \mathcal{K}^{n}$, which gives the $i$-dimensional volume of the projection of $K$ on each $i$-dimensional subspace. Here $i \in \mathbb{N}$ and $1 \leq i \leq n-1$, and the special cases $i=1$ and $i=n-1$ are usually called the width function and brightness function, respectively. The methods and results for this sort of inverse problem form part of the powerful Brunn-Minkowski theory. For example, a classical result of Aleksandrov (see

\footnotetext{
* The first author was supported in part by NSF Grant DMS 9501289. The third author was supported by the Italian Research Council (CNR).
} 
Theorem 3.3.6 of [5]) states:

(1) For each $i \in \mathbb{N}, 1 \leq i \leq n-1$, any centered convex body in $\mathbb{E}^{n}$ is determined in $\mathcal{K}_{c}^{n}$ by its $i$ th projection function.

Here $\mathcal{K}_{c}^{n}$ denotes the class of centered convex bodies; see Section 2 for definitions of unexplained terms. Recently there has been considerable interest in the determination of a convex body in the class $\mathcal{K}^{n}$ of all convex bodies in $\mathbb{E}^{n}$. The main results are as follows (note that a projection function cannot distinguish a convex body $K$ from any of its translates or its reflection $-K$ in the origin):

(2) ([9], from a result in [3].) Centered convex bodies in $\mathbb{E}^{n}$ are determined in $\mathcal{K}^{n}$ by their $i$ th projection functions for two different values of $i, 1 \leq i \leq n-1$.

(3) (See, for example, Chapter 3 of [5].) A convex body in $\mathbb{E}^{n}$ is determined in $\mathcal{K}^{n}$, up to translation and reflection in the origin, by its width function if and only if it is irreducible. (A convex body $K$ is irreducible if the equation $K=\triangle C$, where $\triangle C=\frac{1}{2}(C+(-C))$ is the central symmetral of the convex body $C$, implies that $K$ is a translate of $C$; see pp. 123-4 of [5] for more information.) Such bodies must be centrally symmetric, since $\triangle K$ is a centered convex body with the same width function as $K$.

(4) ([7]; see also [1].) A convex body in $\mathbb{E}^{n}$ is determined in $\mathcal{K}^{n}$, up to translation and reflection in the origin, by its brightness function if and only if it is a parallelotope.

(5) [19] When $1 \leq i<n-1$, most centered convex bodies in $\mathbb{E}^{n}$ are determined in $\mathcal{K}^{n}$ by their $i$ th projection function.

(6) [2] When $2 \leq i \leq n-2$, most convex bodies in $\mathbb{E}^{n}$ are determined in $\mathcal{K}^{n}$, up to translation and reflection in the origin, by their $i$ th projection function.

(7) ([9], a refinement of earlier work of Campi and of Gardner and Volčič; see also [8] and [10].) A dense set of convex bodies in $\mathcal{K}^{n}$ are not determined in $\mathcal{K}^{n}$, up to translation and reflection in the origin, by their $i$ th projection functions for all $i, 1 \leq i \leq n-1$.

(8) [10] Most convex bodies in $\mathbb{E}^{n}$ are determined in $\mathcal{K}^{n}$, up to translation and reflection in the origin, by their width and brightness functions.

In these results, "most" means most in the sense of Baire category, that is, all except for a set of first category in the space $\mathcal{K}^{n}$ endowed with the Hausdorff metric.

It is remarkable that there is a duality in geometric tomography between results on projections of convex bodies and results on sections of star (rather than convex) bodies. This still unexplained fact has led to the creation, due principally to Lutwak, of an extensive dual Brunn-Minkowksi theory; see [5] and [6] for detailed discussions. For example, dual to Aleksandrov's theorem above is a result of Funk (see Theorem 7.2.6 of [5]) which states:

$\left(1^{\prime}\right)$ For each $i \in \mathbb{N}, 1 \leq i \leq n-1$, any centered star body in $\mathbb{E}^{n}$ is determined in $\mathcal{S}_{c}^{n}$ by its $i$ th section function.

Here $\mathcal{S}_{c}^{n}$ denotes the class of centered star bodies in $\mathbb{E}^{n}$ and the $i$ th section function of a star body $L$ gives the $i$-dimensional volume of the section of $L$ by each $i$-dimensional subspace. The special case $i=n-1$ is simply called the section function. 
For determination of a star body in the class $\mathcal{S}^{n}$ of all star bodies in $\mathbb{E}^{n}$, the result dual to (2) above is also known:

$\left(2^{\prime}\right)$ (Theorem 5.9 of [8] or Theorem 7.2.15 of [5]; see also Corollary 4.4 below.) Centered star bodies in $\mathbb{E}^{n}$ are determined in $\mathcal{S}^{n}$ by their $i$ th section functions for two different values of $i, 1 \leq i \leq n-1$.

The main purpose of this paper is to continue the attempt to understand this duality. Corresponding to (3)-(6) above for projections, a simple argument yields the following result:

(3') (Corollary 4.2) When $1 \leq i \leq n-1$, no star body in $\mathbb{E}^{n}$ is determined in $\mathcal{S}^{n}$, up to reflection in the origin, by its $i$ th section function.

We prove the following result corresponding to (7) and (8) above:

(4') (Corollary 4.9) The set of star bodies in $\mathbb{E}^{n}$ that are determined in $\mathcal{S}^{n}$, up to reflection in the origin, by their $i$ th section functions for all $i, 1 \leq i \leq n-1$, is nowhere dense.

We see from the results obtained in this paper that there is a sharp contrast between the situation for projections and that for sections. This is interesting because it indicates possible limitations on the duality referred to above.

Although the duality makes it more natural to study sections of star bodies, convex bodies retain a wide general interest and we also consider in the last section the determination of convex bodies by section functions. In contrast to (4) and (5) above we have, somewhat surprisingly:

$\left(5^{\prime}\right)$ (Theorem 5.1) No centered convex body in $\mathbb{E}^{2}$ is determined in $\mathcal{K}_{o}^{2}$ by its section function.

Here $\mathcal{K}_{o}^{n}$ is the subclass of $\mathcal{K}^{n}$ whose members contain the origin in their interiors. This theorem also shows that the results of [4] on determination of convex polygons in the class of convex polygons do not extend to their determination in the class of all convex bodies.

In Theorem 5.3 we show that a dense set of convex bodies in $\mathcal{K}_{o}^{n}$, those with everywhere positive Gaussian curvature, are not determined in $\mathcal{K}_{o}^{n}$, up to reflection in the origin, by their $i$ th section functions. Despite this, the question of whether most convex bodies in $\mathbb{E}^{n}$ are determined in $\mathcal{K}_{o}^{n}$, up to reflection in the origin, by their section functions remains open. One has to work quite hard to find any member of $\mathcal{K}_{o}^{n}$ that is determined in this sense; in Theorem 5.4 we show that examples are provided by triangles whose centroids lie at the origin. This result and Fig. 2 are due to the second author.

\section{Definitions and Preliminaries}

If $A$ is a set, we write int $A$, bd $A$, and conv $A$ for the interior, boundary, and convex hull of $A$, respectively. We denote the origin, unit sphere, and closed unit ball in $n$ dimensional Euclidean space $\mathbb{E}^{n}$ by $o, S^{n-1}$, and $B$, respectively. If $u \in S^{n-1}$, we denote by $u^{\perp}$ the $(n-1)$-dimensional subspace orthogonal to $u$. For $1 \leq i \leq n-1$, we denote 
the Grassmann manifold of $i$-dimensional subspaces in $\mathbb{E}^{n}$ by $\mathcal{G}(n, i)$. If $1 \leq i \leq n$, we write $\lambda_{i}$ for Lebesgue $i$-dimensional measure in $\mathbb{E}^{n}$ (which we identify with $i$-dimensional Hausdorff measure in $\mathbb{E}^{n}$ ), and $\kappa_{n}=\lambda_{n}(B)$.

We say that a set is centered if it is centrally symmetric, with center at the origin. A convex body is a compact convex set with nonempty interior. The class of convex bodies in $\mathbb{E}^{n}$ is denoted by $\mathcal{K}^{n}$, and $\mathcal{K}_{c}^{n}, \mathcal{K}_{o}^{n}$ signify the subclasses consisting of centered convex bodies and convex bodies containing the origin in their interiors, respectively.

A set $L$ is star-shaped at the origin if every line through the origin that meets $L$ does so in a (possibly degenerate) closed line segment. If $L$ is a set which is star-shaped at the origin, its radial function $\rho_{L}$ is defined, for all $u \in S^{n-1}$ such that the line through the origin parallel to $u$ intersects $L$, by

$$
\rho_{L}(u)=\max \{c: c u \in L\} .
$$

We denote by $\mathcal{S}^{n}$ the set of all nonempty compact sets which are star-shaped at the origin and whose radial functions are defined and continuous on $S^{n-1}$. Each member of $\mathcal{S}^{n}$ contains the origin and, with the single exception of the singleton set $\{o\}$, is a body in the sense that it is the closure of its interior. Let $\mathcal{S}_{c}^{n}$ be the set of all centered members of $\mathcal{S}^{n}$ and let $\mathcal{S}_{o}^{n}$ denote the set of members of $\mathcal{S}^{n}$ that contain the origin in their interiors.

In this paper we call members of $\mathcal{S}^{n}$ star bodies. We note, however, that there are different definitions of this term in the recent literature. In the work of Lutwak [16], [17] and others, the term implies membership in the smaller class $\mathcal{S}_{o}^{n}$. Another definition, introduced by two of the present authors in [8], allows for certain sets not containing the origin. In addition to these variants, Klain [14] considers the class of sets $L$ star-shaped at the origin in $\mathbb{E}^{n}$ and such that $\rho_{L}$ is an $L^{n}$ function on $S^{n-1}$; such sets are called $L^{n}$-stars.

Observe that a convex body is a star body if and only if it contains the origin and its boundary does not contain a line segment containing the origin.

We now define the $i$-chord functions of a star body. (See Chapter 6 of [5] for extensive comments and history concerning this much-studied concept.) Let $i>0$ be a real number and let $L$ be a star body in $\mathbb{E}^{n}$. The $i$-chord function $\rho_{i, L}$ of $L$ is defined by

$$
\rho_{i, L}(u)=\rho_{L}(u)^{i}+\rho_{L}(-u)^{i},
$$

for $u \in S^{n-1}$. When $i=1$, this gives the length of chords of $L$ through the origin, and for this reason, $\rho_{1, L}$ is sometimes also called the $X$-ray of $L$ at the origin. Another generalization of the latter is the $i$ th section function of $L$, defined for integer values of $i$ with $1 \leq i \leq n-1$ by $\lambda_{i}(L \cap S)$, for $S \in \mathcal{G}(n, i)$. The following known result (see Theorem 7.2.3 and Remark 7.2.5 of [5], and compare Theorem 3.1 of [8]) connects these definitions.

Proposition 2.1. Let $i \in \mathbb{N}$ and $1 \leq i \leq n-1$. Two star bodies in $\mathbb{E}^{n}$ have equal $i$-chord functions if and only if they have equal ith section functions.

The following result (see Theorem 5.2 of [8] or Theorem 6.2.16 of [5]) will also be useful in what follows. 
Proposition 2.2. Let $i$ and $j$ be distinct positive real numbers. Two star bodies $L$ and $M$ in $\mathbb{E}^{n}$ have equal $i$-chord functions and equal $j$-chord functions if and only if

$$
\left\{\rho_{L}(u), \rho_{L}(-u)\right\}=\left\{\rho_{M}(u), \rho_{M}(-u)\right\},
$$

for all $u \in S^{n-1}$.

\section{Metrics for Star Bodies}

The distance between nonempty compact sets $C$ and $D$ can be measured by means of the Hausdorff metric, defined by

$$
\delta(C, D)=\min \{\varepsilon>0: C \subset D+\varepsilon B \text { and } D \subset C+\varepsilon B\} .
$$

For star bodies, however, the radial metric $\tilde{\delta}$ is in many ways more natural. This is defined by setting

$$
\tilde{\delta}(L, M)=\max _{u \in S^{n-1}}\left|\rho_{L}(u)-\rho_{M}(u)\right|,
$$

for star bodies $L$ and $M$. With this metric, $\mathcal{S}^{n}$ is a complete metric space and hence a Baire space. It is easy to see that $\mathcal{S}_{o}^{n}$ is an open and dense subset of $\mathcal{S}^{n}$.

Goodey and Weil [11] prove that convergence in the Hausdorff metric does not imply convergence in the radial metric. It is easy to see that $\left(\mathcal{S}^{n}, \delta\right)$ is not a complete metric space. Our preference for the radial metric here is a consequence of the following stronger result.

Theorem 3.1. The set $\mathcal{S}^{n}$, when endowed with the Hausdorff metric, is not a Baire space.

Proof. For each $k \in \mathbb{N}$, let

$$
\mathcal{G}_{k}=\left\{L \in \mathcal{S}^{n}: \lambda_{n-1}\left(\left\{u \in S^{n-1}: \rho_{L}(u) \geq \frac{1}{k}\right\}\right)<\frac{1}{k}\right\} .
$$

We claim that $\mathcal{G}_{k}$ is open in $\left(\mathcal{S}^{n}, \delta\right)$. To see this, suppose that $L \in \mathcal{G}_{k}$. For $m \in \mathbb{N}$, let $L_{m}=L+(1 / m) B$; it is easy to see that $L_{m} \in \mathcal{S}^{n}$ and $\bigcap_{m} L_{m}=L$. The sets

$$
E_{m}=\left\{u \in S^{n-1}: \rho_{L_{m}}(u) \geq \frac{1}{k}\right\}
$$

decrease with intersection

$$
E=\left\{u \in S^{n-1}: \rho_{L}(u) \geq \frac{1}{k}\right\}
$$

It follows that $\lambda_{n-1}\left(E_{m}\right)$ decreases with limit $\lambda_{n-1}(E)$, so there is an $m_{0}$ such that 
$\lambda_{n-1}\left(E_{m_{0}}\right)<1 / k$. Let $M \in \mathcal{S}^{n}$ be such that $\delta(L, M)<1 / m_{0}$. Then $M \subset L_{m_{0}}$, so

$$
\lambda_{n-1}\left(\left\{u \in S^{n-1}: \rho_{M}(u) \geq \frac{1}{k}\right\}\right)<\lambda_{n-1}\left(E_{m_{0}}\right)<\frac{1}{k}
$$

which shows that $M \in \mathcal{G}_{k}$, proving the claim.

Let $L \in \mathcal{S}^{n}$ and let $\varepsilon>0$. Then there is a finite subset $F$ of $S^{n-1}$ such that if

$$
C=\bigcup\left\{\left[o, \rho_{L}(u) u\right]: u \in F\right\},
$$

then $C$ is a finite union of line segments with $\delta(C, L)<\varepsilon / 2$. Clearly, there is also an $M \in \mathcal{G}_{k}$ such that $\delta(M, C)<\varepsilon / 2$. Then $\delta(L, M)<\varepsilon$, so $\mathcal{G}_{k}$ is dense in $\left(\mathcal{S}^{n}, \delta\right)$.

Let $L$ be any member of $\mathcal{S}^{n}$ other than $\{o\}$. Then there is a $u_{0} \in S^{n-1}$ such that $\rho_{L}\left(u_{0}\right)>1 / m$ for some $m \in \mathbb{N}$. Choose $k \in \mathbb{N}$ with $k \geq m$ and

$$
\lambda_{n-1}\left(\left\{u \in S^{n-1}: \rho_{L}(u) \geq \frac{1}{m}\right\}\right) \geq \frac{1}{k} .
$$

Then $L \notin \mathcal{G}_{k}$, so $\bigcap_{k} \mathcal{G}_{k}=\{o\}$ is not dense in $\left(\mathcal{S}^{n}, \delta\right)$. This proves the theorem.

\section{Determination of Star Bodies}

Theorem 4.1. Let $i>0$. No star body in $\mathbb{E}^{n}$ is determined in $\mathcal{S}^{n}$, up to reflection in the origin, by its $i$-chord function.

Proof. Let $L \in \mathcal{S}^{n}$. Choose $u_{0} \in S^{n-1}$ with $\rho_{L}\left(u_{0}\right)>0$, and let $U$ be any open cap containing $u_{0}$ and contained in a hemisphere in $S^{n-1}$ such that $\rho_{L}(u)>0$ for all $u \in U$. Let $f \in C\left(S^{n-1}\right)$ be such that $0<f(u)<\rho_{L}(u)$ for $u \in U, f(u)=0$ for $u \notin U$, and

$$
f\left(u_{0}\right) \neq \rho_{L}\left(u_{0}\right)-\rho_{L}\left(-u_{0}\right) .
$$

Let

$$
g(u)=\left(\rho_{L}(u)^{i}+\rho_{L}(-u)^{i}-\left(\rho_{L}(u)-f(u)\right)^{i}\right)^{1 / i}-\rho_{L}(-u),
$$

$u \in U$. Define $M \in \mathcal{S}^{n}$ by $\rho_{M}(u)=\rho_{L}(u)-f(u)$ if $u \in U, \rho_{M}(u)=\rho_{L}(u)+g(-u)$ if $u \in-U$, and $\rho_{M}(u)=\rho_{L}(u)$ otherwise. It is easy to check that $\rho_{M} \in C\left(S^{n-1}\right)$, that $L$ and $M$ have equal $i$-chord functions, and that $\rho_{M}\left(u_{0}\right) \neq \rho_{L}\left( \pm u_{0}\right)$, as required.

Corollary 4.2. Let $i \in \mathbb{N}$ and $1 \leq i \leq n-1$. No star body in $\mathbb{E}^{n}$ is determined in $\mathcal{S}^{n}$, up to reflection in the origin, by its $i$ th section function.

Proof. This follows immediately from Proposition 2.1 and the previous theorem.

Theorem 4.3. A star body in $\mathbb{E}^{n}$ is determined in $\mathcal{S}^{n}$, up to reflection in the origin, by its $i$-chord functions for two different values of $i$ if and only if the set

$$
V_{L}=\left\{u \in S^{n-1}: \rho_{L}(u)>\rho_{L}(-u)\right\}
$$

is connected. 
Proof. Suppose that $V_{L}$ is not connected and let $U$ be one of its components. By Proposition 2.2 the star body $M$ with radial function

$$
\rho_{M}(u)= \begin{cases}\rho_{L}(-u) & \text { if } u \in U \cup(-U), \\ \rho_{L}(u) & \text { otherwise, }\end{cases}
$$

has all its $i$-chord functions equal to those of $L$, but $M \neq \pm L$.

Conversely, suppose that $V_{L}$ is connected and that $M$ has its $i$-chord functions equal to those of $L$ for two different values of $i$, but $M \neq \pm L$. By Proposition 2.2,

$$
\left\{\rho_{L}(u), \rho_{L}(-u)\right\}=\left\{\rho_{M}(u), \rho_{M}(-u)\right\} .
$$

This implies that there exist $u_{1}$ and $u_{2}$, where we may assume $u_{1}, u_{2} \in V_{L}$, such that

$$
\rho_{M}\left(u_{1}\right)=\rho_{L}\left(u_{1}\right) \neq \rho_{M}\left(-u_{1}\right)=\rho_{L}\left(-u_{1}\right)
$$

and

$$
\rho_{M}\left(u_{2}\right)=\rho_{L}\left(-u_{2}\right) \neq \rho_{M}\left(-u_{2}\right)=\rho_{L}\left(u_{2}\right) \text {. }
$$

Then we have

$$
\rho_{M}\left(u_{1}\right)>\rho_{M}\left(-u_{1}\right)
$$

and

$$
\rho_{M}\left(u_{2}\right)<\rho_{M}\left(-u_{2}\right) .
$$

Since $V_{L}$ is connected and $\rho_{M}$ is continuous, there exists $u_{0} \in V_{L}$ such that $\rho_{M}\left(u_{0}\right)=$ $\rho_{M}\left(-u_{0}\right)$ and therefore $\rho_{L}\left(u_{0}\right)=\rho_{L}\left(-u_{0}\right)$, a contradiction.

Corollary 4.4. A centered star body in $\mathbb{E}^{n}$ is determined in $\mathcal{S}^{n}$ by its $i$ th section functions for two different values of $i$.

Proof. This follows directly from Proposition 2.1 and the previous theorem.

Let $M$ be a star body. For the remainder of this section we continue to use the notation of Theorem 4.3, so that

$$
V_{M}=\left\{u \in S^{n-1}: \rho_{M}(u)>\rho_{M}(-u)\right\} .
$$

Denote by $\mathcal{M}$ the set of $M \in \mathcal{S}_{o}^{n}$ such that there exist disjoint open components $U_{1}, U_{2}$ of $V_{M}$ which are separated by $-V_{M}$, that is, $U_{1}$ and $U_{2}$ are contained in disjoint components of $S^{n-1} \backslash\left(-V_{M}\right)$.

The following lemma provides a useful characterization of $\mathcal{M}$.

Lemma 4.5. A star body $M \in \mathcal{S}_{o}^{n}$ belongs to $\mathcal{M}$ if and only if there exist open components $U_{1}$ and $U_{2}$ of $V_{M}$ and an $a>0$ such that

$$
W_{M}(a)=\left\{u \in S^{n-1}: \rho_{M}(u)<\rho_{M}(-u)-a\right\}
$$

separates $U_{1}$ from $U_{2}$. 
Proof. Let $M \in \mathcal{S}_{o}^{n}$. It is clear that if the stated condition holds, then $M \in \mathcal{M}$.

Conversely, suppose that $M \in \mathcal{M}$. Let $U_{1}$ and $U_{2}$ be open components of $V_{M}$ contained in disjoint components of $S^{n-1} \backslash\left(-V_{M}\right)$. For $k \in \mathbb{N}$, the sets $W_{M}(1 / k)$ are open in $S^{n-1}$ and increase with union $-V_{M}$. Then $S^{n-1} \backslash W_{M}(1 / k)$ is closed and contains $U_{1}$ and $U_{2}$, for each $k \in \mathbb{N}$. Suppose that, for all $k$, both $U_{1}$ and $U_{2}$ are contained in the same (compact) component $C_{k}$ of $S^{n-1} \backslash W_{M}(1 / k)$. Then $C=\bigcap_{k} C_{k}$ is a component of $S^{n-1} \backslash\left(-V_{M}\right)$ (see, for example, Theorem 5 of Section 47 in [15]) that contains both $U_{1}$ and $U_{2}$, a contradiction. Therefore there is a $k_{0}$ such that $W_{M}\left(1 / k_{0}\right)$ separates $U_{1}$ from $U_{2}$. Letting $a=1 / k_{0}$, we have proved the lemma.

Lemma 4.6. The set $\mathcal{M}$ is open in $\mathcal{S}_{o}^{n}$.

Proof. Let $M \in \mathcal{M}$ and let $U_{1}, U_{2}, a>0$, and $W_{M}(a)$ be as in Lemma 4.5. Let $a_{j}=\max _{u \in U_{j}}\left(\rho_{M}(u)-\rho_{M}(-u)\right), j=1,2$, and let $0<\varepsilon<\min \left\{a_{1}, a_{2}, a\right\} / 2$. Suppose that $L \in \mathcal{S}_{o}^{n}$ and $\tilde{\delta}(L, M)<\varepsilon$, which implies that

$$
\rho_{M}(u)-\varepsilon<\rho_{L}(u)<\rho_{M}(u)+\varepsilon,
$$

for all $u \in S^{n-1}$. Let $u_{j} \in U_{j}$ be such that $\rho_{M}\left(u_{j}\right)=a_{j}, j=1,2$. Then

$$
\rho_{L}\left(u_{j}\right)-\rho_{L}\left(-u_{j}\right)>\rho_{M}\left(u_{j}\right)-\varepsilon-\rho_{M}\left(-u_{j}\right)-\varepsilon=a_{j}-2 \varepsilon>0,
$$

so $u_{j} \in V_{L}, j=1,2$. Let $U_{j}^{\prime}$ be the component of $V_{L}$ that contains $u_{j}, j=1,2$, and let $a^{\prime}=a-2 \varepsilon$. If $u \in W_{M}(a)$, then

$$
\rho_{L}(u)-\rho_{L}(-u)<\rho_{M}(u)+\varepsilon-\rho_{M}(-u)+\varepsilon<-a+2 \varepsilon=-a^{\prime},
$$

so $u \in W_{L}\left(a^{\prime}\right)$. Therefore $W_{M}(a) \subset W_{L}\left(a^{\prime}\right)$ and since $U_{j}^{\prime} \cap W_{L}\left(a^{\prime}\right)=\emptyset$ and $U_{j} \cap U_{j}^{\prime} \neq \emptyset$, $j=1,2, W_{L}\left(a^{\prime}\right)$ separates $U_{1}^{\prime}$ and $U_{2}^{\prime}$. So $L \in \mathcal{M}$, as required.

Lemma 4.7. The set $\mathcal{M}$ is dense in $\mathcal{S}_{o}^{n}$.

Proof. Suppose that $L \in \mathcal{S}_{o}^{n}$, and let $0<\varepsilon<\min _{u \in S^{n-1}} \rho_{L}(u)$. From the continuity of $\rho_{L}$ if follows that for each $\varepsilon>0$ there is an open cap $C$ contained in a hemisphere of $S^{n-1}$ such that

$$
\rho_{L}(-u)-\frac{\varepsilon}{2}<\rho_{L}(u)<\rho_{L}(-u)+\frac{\varepsilon}{2}
$$

for all $u \in C$. Choose distinct points $u_{1}$ and $u_{2}$ in $C$. Let $C_{1}$ and $C_{2}$ be closed caps centered at $u_{1}$ and contained in $C$, with the radius of $C_{1}$ greater than that of $C_{2}$, and such that $u_{2} \notin C_{1}$. Let $A=$ relint $C_{1} \backslash C_{2}$; then $A$ is an annular region in $S^{n-1}$ centered at $u_{1}$.

Let $f(u)$ be a continuous function on $S^{n-1}$ such that $|f(u)| \leq \varepsilon$ for all $u \in S^{n-1}$, $f\left(u_{j}\right)=\varepsilon, j=1,2, f(u)=-\varepsilon$ for $u \in A$, and $f(u)=0$ if $u \notin C$. Let $M$ be the star body defined by

$$
\rho_{M}(u)=\rho_{L}(u)+f(u) .
$$

Then

$$
\rho_{M}\left(u_{j}\right)=\rho_{L}\left(u_{j}\right)+\varepsilon>\rho_{L}\left(-u_{j}\right)+\frac{\varepsilon}{2}=\rho_{M}\left(-u_{j}\right)+\frac{\varepsilon}{2}>\rho_{M}\left(-u_{j}\right),
$$


so $u_{j} \in V_{M}$, for $j=1,2$. On the other hand, for $u \in A$ we have

$$
\rho_{M}(u)=\rho_{L}(u)-\varepsilon<\rho_{L}(-u)-\frac{\varepsilon}{2}=\rho_{M}(-u)-\frac{\varepsilon}{2},
$$

so $A \subset W_{M}(\varepsilon / 2)$. If $U_{j}$ is the component of $V_{M}$ containing $u_{j}, j=1,2$, then $U_{1}$ and $U_{2}$ are separated by $A$ and so also $W_{M}(\varepsilon / 2)$. Therefore $M \in \mathcal{M}$ and $\tilde{\delta}(L, M)=\varepsilon$.

Lemma 4.8. If $M \in \mathcal{M}$, there exists an $M^{\prime} \in \mathcal{M}$ such that $M^{\prime} \neq \pm M$ and yet $M$ and $M^{\prime}$ have equal $i$-chord functions for all $i>0$.

Proof. Let $M \in \mathcal{M}$ and let $U_{1}, U_{2}, a>0$, and $W_{M}(a)$ be as in Lemma 4.5. Define $M^{\prime}$ by

$$
\rho_{M^{\prime}}(u)=\left\{\begin{array}{lll}
\rho_{M}(-u) & \text { if } & u \in U_{1} \cup\left(-U_{1}\right), \\
\rho_{M}(u) & \text { if } \quad u \in S^{n-1} \backslash\left(U_{1} \cup\left(-U_{1}\right)\right) .
\end{array}\right.
$$

We claim that $\rho_{M^{\prime}}$ is continuous. It is obviously continuous at each $u \in U_{1} \cup\left(-U_{1}\right)$ and at each point in the relative interior of $S^{n-1} \backslash\left(U_{1} \cup\left(-U_{1}\right)\right)$. If $u_{0}$ belongs to the common relative boundary of these sets, then

$$
\rho_{M^{\prime}}\left(u_{0}\right)=\rho_{M}\left(u_{0}\right)=\rho_{M}\left(-u_{0}\right)
$$

For each $\varepsilon>0$ there exists a neighborhood $U$ of $u_{0}$ such that $\left|\rho_{M}\left(u_{0}\right)-\rho_{M}(u)\right|<\varepsilon$ and $\left|\rho_{M}\left(-u_{0}\right)-\rho_{M}(-u)\right|<\varepsilon$ for each $u \in U$. If $u \in U \cap\left(U_{1} \cup\left(-U_{1}\right)\right)$, we have

$$
\left|\rho_{M^{\prime}}\left(u_{0}\right)-\rho_{M^{\prime}}(u)\right|=\left|\rho_{M}\left(-u_{0}\right)-\rho_{M}(-u)\right|<\varepsilon,
$$

while if $u \in U \backslash\left(U_{1} \cup\left(-U_{1}\right)\right)$, then

$$
\left|\rho_{M^{\prime}}\left(u_{0}\right)-\rho_{M^{\prime}}(u)\right|=\left|\rho_{M}\left(u_{0}\right)-\rho_{M}(u)\right|<\varepsilon,
$$

and this proves the claim.

Since

$$
\left\{\rho_{M}(u), \rho_{M}(-u)\right\}=\left\{\rho_{M^{\prime}}(u), \rho_{M^{\prime}}(-u)\right\}
$$

for all $u \in S^{n-1}$, Proposition 2.2 implies that $M$ and $M^{\prime}$ have equal $i$-chord functions for all $i>0$. Moreover, since $\rho_{M}(u)>\rho_{M}(-u)=\rho_{M^{\prime}}(u)$ when $u \in U_{1}$, we have $M \neq M^{\prime}$, and since $\rho_{M}(u)>\rho_{M}(-u)=\rho_{M^{\prime}}(-u)$ when $u \in U_{2}$, we also have $M \neq-M^{\prime}$.

Corollary 4.9. The set of star bodies in $\mathbb{E}^{n}$ that are determined in $\mathcal{S}^{n}$, up to reflection in the origin, by their $i$ th section functions for all $i, 1 \leq i \leq n-1$, is nowhere dense.

Proof. By Lemmas 4.6, 4.7, and 4.8, there is an open dense subset of $\mathcal{S}_{o}^{n}$, and hence an open dense subset of $\mathcal{S}^{n}$, consisting of star bodies not determined, up to reflection in the origin, by their $i$-chord functions for all $i>0$. The result now follows from Proposition 2.2. 
We know from Lemma 4.8 that the set of star bodies in $\mathcal{S}_{o}^{n}$ that are not determined in $\mathcal{S}^{n}$, up to reflection in the origin, by their $i$ th section functions for two different values of $i$, contains the open set $\mathcal{M}$, dense in $\mathcal{S}_{o}^{n}$. Despite this, we have the following result.

Theorem 4.10. The set of all star bodies in $\mathcal{S}_{o}^{n}$ that are not determined in $\mathcal{S}^{n}$, up to reflection in the origin, by their $i$ th section functions for two different values of $i$, is not open.

Proof. Let $L^{\prime}$ be the planar star body such that

$$
\rho_{L^{\prime}}(\theta)= \begin{cases}1+\sin ^{2} 4 \theta & \text { if } \quad 0 \leq \theta \leq \pi \\ 1 & \text { if } \pi \leq \theta \leq 2 \pi\end{cases}
$$

Since $L^{\prime}$ is symmetric with respect to the $y$-axis, we can rotate a copy of $L^{\prime}$ in the $\left(x_{1}, x_{n}\right)$-plane in $\mathbb{E}^{n}$ about the $x_{n}$-axis to obtain a star body $L$ in $\mathbb{E}^{n}$.

By Theorem 4.3, $L$ is not determined, up to reflection in the origin, by its $i$ th section functions for two different values of $i$, since $V_{L}$ has components $\left\{u \in S^{n-1}: 0<u \cdot u_{0}<\right.$ $1 / \sqrt{2}\}$ and $\left\{u \in S^{n-1}: 1 / \sqrt{2}<u \cdot u_{0}<1\right\}$, where $u_{0}=(0, \ldots, 0,1)$. Let $\varepsilon>0$, and define a planar star body $M^{\prime}$ by

$$
\rho_{M^{\prime}}(\theta)= \begin{cases}1+\sin ^{2} 4 \theta+\varepsilon(2 / \pi)^{2} \theta(\pi-\theta) & \text { if } \quad 0 \leq \theta \leq \pi, \\ 1 & \text { if } \pi \leq \theta \leq 2 \pi\end{cases}
$$

Note that $\tilde{\delta}\left(L^{\prime}, M^{\prime}\right)=\varepsilon$. Since $M^{\prime}$ is symmetric with respect to the $y$-axis, we can rotate a copy of $M^{\prime}$ in the $\left(x_{1}, x_{n}\right)$-plane in $\mathbb{E}^{n}$ about the $x_{n}$-axis to obtain a star body $M$ in $\mathbb{E}^{n}$ such that $\tilde{\delta}(L, M)=\varepsilon$. Since

$$
V_{M}=\left\{u \in S^{n-1}: u \cdot u_{0}>0\right\}
$$

Theorem 4.3 implies that $M$ is determined, up to reflection in the origin, by its $i$ th section functions for two different values of $i$.

\section{Determination of Convex Bodies}

Theorem 5.1. Let $i>0$ and suppose that $K$ is a centered convex body in $\mathbb{E}^{2}$. Then $K$ is not determined in $\mathcal{K}_{o}^{2}$ by its $i$-chord function (and hence not by its section function or $X$-ray at the origin).

Proof. By rotating $K$, if necessary, we may suppose that it is strictly convex at the point $v$ where its boundary bd $K$ meets the positive $x$-axis; since $K$ is centered, it is also strictly convex at $-v$. Let $w$ be a point on the positive $x$-axis with $w \notin K$, and let $p_{j}$, $j=1,2$, be the points where the tangents to $K$ through $w$ meet bd $K$. We assume that $w$ is sufficiently close to bd $K$ to ensure that if $u$ is a unit vector in the direction of a point $q$ on $\left[p_{j}, w\right], j=1,2$, then

$$
2^{1 / i} \rho_{K}(u)>\|q\| .
$$


Let $L \in \mathcal{S}_{o}^{2}$ be the star body with the same $i$-chord functions as $K$, such that bd $L$ is obtained from bd $K$ by replacing the arc in bd $K$ from $p_{1}$ to $p_{2}$ containing $v$ by the line segments $\left[p_{j}, w\right]$, for $j=1,2$, and the arc in bd $K$ from $-p_{1}$ to $-p_{2}$ containing $-v$ by suitable arcs $P_{j}$ joining $-p_{j}$ and a point $z \in K$ on the negative $x$-axis. The body $L$ is defined by

$$
\rho_{L}(u)^{i}+\rho_{L}(-u)^{i}=\rho_{K}(u)^{i}+\rho_{K}(-u)^{i}=2 \rho_{K}(u)^{i},
$$

for all $u \in S^{1}$.

The body $L$ is in general not convex (see Fig. 1 for a special case when it is), but we claim that the $\operatorname{arcs} P_{j}, j=1,2$, are convex with respect to the origin. To see this, fix $j$ and let $u_{1}$ and $u_{2}$ be unit vectors in the direction of points on $\left[p_{j}, w\right]$. The convexity of $K$ implies (see Lemma 5.1.4 of [5]) that

$$
\rho_{K}\left(u_{1}+u_{2}\right)^{-1} \leq \rho_{K}\left(u_{1}\right)^{-1}+\rho_{K}\left(u_{2}\right)^{-1}
$$

and the linearity of bd $L$ along $\left[p_{j}, w\right]$ yields

$$
\rho_{L}\left(u_{1}+u_{2}\right)^{-1}=\rho_{L}\left(u_{1}\right)^{-1}+\rho_{L}\left(u_{2}\right)^{-1} \text {. }
$$

For $p<0$, Minkowski's inequality states that

$$
\left(\left(a_{1}+b_{1}\right)^{p}+\left(a_{2}+b_{2}\right)^{p}\right)^{1 / p} \geq\left(a_{1}^{p}+a_{2}^{p}\right)^{1 / p}+\left(b_{1}^{p}+b_{2}^{p}\right)^{1 / p},
$$

for $a_{k}>0$ and $b_{k}>0, k=1,2$; see (B.4), p. 367 of [5] or Section 2.11 of [13]. Letting $c_{k}=a_{k}+b_{k}, k=1,2$, and $p=-1 / i$, we obtain

$$
\left(c_{1}^{-1 / i}+c_{2}^{-1 / i}\right)^{-i}-\left(b_{1}^{-1 / i}+b_{2}^{-1 / i}\right)^{-i} \geq\left(\left(c_{1}-b_{1}\right)^{-1 / i}+\left(c_{2}-b_{2}\right)^{-1 / i}\right)^{-i},
$$

for $c_{k}>b_{k}>0, k=1,2$.

By (1), we have $2^{1 / i} \rho_{K}\left(u_{k}\right)>\rho_{L}\left(u_{k}\right), k=1,2$. Then we can apply (5) with $b_{k}=$ $\rho_{L}\left(u_{k}\right)^{i}$ and $c_{k}=2 \rho_{K}\left(u_{k}\right)^{i}, k=1,2$, together with (2)-(4), to obtain

$$
\begin{aligned}
\rho_{L}\left(-u_{1}-u_{2}\right)^{-1}= & \left(2 \rho_{K}\left(u_{1}+u_{2}\right)^{i}-\rho_{L}\left(u_{1}+u_{2}\right)^{i}\right)^{-1 / i} \\
\leq & \left(\left(2^{-1 / i} \rho_{K}\left(u_{1}\right)^{-1}+2^{-1 / i} \rho_{K}\left(u_{2}\right)^{-1}\right)^{-i}\right. \\
& \left.-\left(\rho_{L}\left(u_{1}\right)^{-1}+\rho_{L}\left(u_{2}\right)^{-1}\right)^{-i}\right)^{-1 / i} \\
= & \left(\left(\left(2 \rho_{K}\left(u_{1}\right)^{i}\right)^{-1 / i}+\left(2 \rho_{K}\left(u_{2}\right)^{i}\right)^{-1 / i}\right)^{-i}\right. \\
& \left.\quad-\left(\left(\rho_{L}\left(u_{1}\right)^{i}\right)^{-1 / i}+\left(\rho_{L}\left(u_{2}\right)^{i}\right)^{-1 / i}\right)^{-i}\right)^{-1 / i} \\
\leq & \left(2 \rho_{K}\left(u_{1}\right)^{i}-\rho_{L}\left(u_{1}\right)^{i}\right)^{-1 / i}+\left(2 \rho_{K}\left(u_{2}\right)^{i}-\rho_{L}\left(u_{2}\right)^{i}\right)^{-1 / i} \\
= & \rho_{L}\left(-u_{1}\right)^{-1}+\rho_{L}\left(-u_{2}\right)^{-1} .
\end{aligned}
$$

This shows that the $\operatorname{arc} P_{j}$ is convex and proves the claim.

If $L$ is convex, the proof is finished. Otherwise, let $H=$ conv $L$. Then there are points $q_{j} \in P_{j}, j=1,2$, such that bd $H$ contains the line segment $\left[q_{1}, q_{2}\right]$ but otherwise agrees with bd $L$. 
Let $M \in \mathcal{S}_{o}^{2}$ be the star body defined by

$$
\rho_{M}(u)^{i}+\rho_{H}(-u)^{i}=\rho_{L}(u)^{i}+\rho_{L}(-u)^{i}=2 \rho_{K}(u)^{i},
$$

for all $u \in S^{1}$, where the last equality is a consequence of (2). The points in bd $M$ in the direction of $u \in S^{1}$ such that $-u$ is in the direction of a point in $\left[q_{1}, q_{2}\right]$ form an arc $R$ joining points $r_{1}$ and $r_{2}$, say, with $r_{j} \in\left[p_{j}, w\right]$.

The $\operatorname{arc} R$ is convex with respect to the origin. The argument that proves this is the same as that for the convexity of the arc $P_{j}$, since if $u_{1}$ and $u_{2}$ are unit vectors in the direction of points on $\left[q_{1}, q_{2}\right]$, we have

$$
\rho_{M}\left(-u_{1}-u_{2}\right)^{-1}=\left(2 \rho_{K}\left(u_{1}+u_{2}\right)^{i}-\rho_{H}\left(u_{1}+u_{2}\right)^{i}\right)^{-1 / i},
$$

and bd $H$ is linear along $\left[q_{1}, q_{2}\right]$.

Finally, we let $K^{\prime}$ be the body whose boundary contains the $\operatorname{arc} R$ but otherwise agrees with bd $H$. By construction, $K^{\prime} \in \mathcal{K}_{o}^{2}$ and $K^{\prime}$ has the same $i$-chord function as $K$, so the proof is complete.

Figure 1 depicts a body $K^{\prime}$ obtained from a centered square $K$ by the construction of the previous theorem with $i=1$. For this example it can be shown that when $w$ is sufficiently close to $v$, as it is in Figure 1, the body $L$ in the proof of Theorem 5.1 is already convex. Figure 1 was generated with Mathematica and therefore gives the precise shape of $K^{\prime}$ up to the tolerances of that package. The existence of such examples is surprising and of independent interest since it would be natural to conjecture that a convex body containing the origin in its interior and having the same X-ray at the origin as a convex polygon must itself be a convex polygon. The example also shows that the results of [4] on determination of convex polygons in the class of convex polygons do not extend to their determination in the class of all convex bodies.

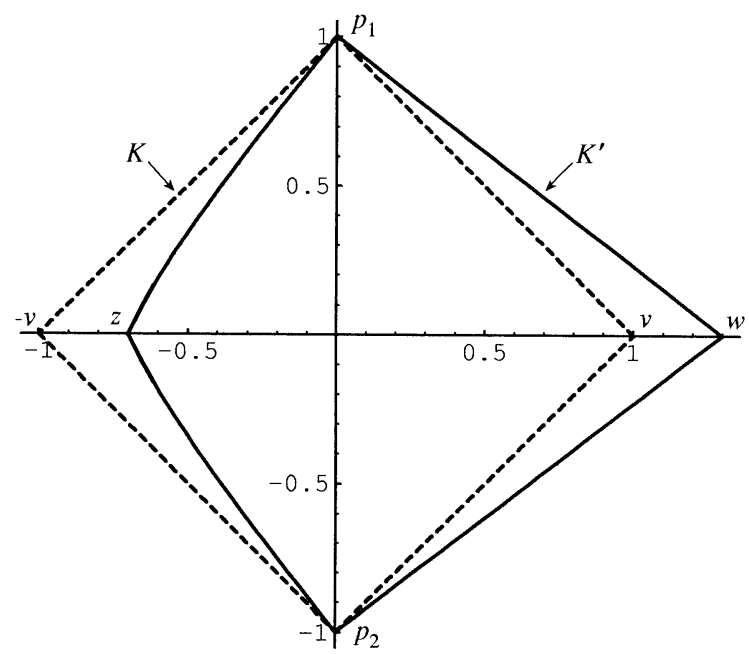

Fig. 1. Convex bodies with the same section function. 
We conjecture that Theorem 5.1 holds in higher dimensions. The following question is also open.

Question 5.2. Let $i \in \mathbb{N}$ and $1 \leq i \leq n-1$. Are most convex bodies in $\mathcal{K}_{o}^{n}$ determined in $\mathcal{K}_{o}^{n}$ by their $i$ th section function?

The next theorem provides an important class of convex bodies in $\mathbb{E}^{n}$ that are not determined in the sense of the previous question.

Theorem 5.3. Let $i>0$ and suppose that $K \in \mathcal{K}_{o}^{n}$ has everywhere positive Gaussian curvature. Then $K$ is not determined in $\mathcal{K}_{o}^{n}$, up to reflection in the origin, by its $i$-chord function, or (if $i \in \mathbb{N}$ and $1 \leq i \leq n-1$ ) by its $i$ th section function.

Proof. Let $U$ be an open cap contained in a hemisphere in $S^{n-1}$ and let $f \in C^{2}\left(S^{n-1}\right)$ be such that $f(u)>0$ if $u \in U$ and $f(u)=0$ if $u \notin U$. Let $c>0$ be such that $c f(u)<\rho_{K}(u)$ if $u \in U$. Define

$$
g_{c}(u)=\left(\rho_{K}(u)^{i}+\rho_{K}(-u)^{i}-\left(\rho_{K}(u)-c f(u)\right)^{i}\right)^{1 / i}-\rho_{K}(-u),
$$

for $u \in U$. Define $L \in \mathcal{S}_{o}^{n}$ by

$$
\rho_{L}(u)= \begin{cases}\rho_{K}(u)-c f(u) & \text { if } u \in U, \\ \rho_{K}(u)+g_{c}(-u) & \text { if } u \in-U, \\ \rho_{K}(u) & \text { otherwise. }\end{cases}
$$

It is easy to check that $\rho_{L} \in C^{2}\left(S^{n-1}\right)$ and that $K$ and $L$ have equal $i$-chord functions (and by Proposition 2.1, if $i \in \mathbb{N}$ and $1 \leq i \leq n-1$, equal $i$ th section functions). We can also choose $u_{0} \in U$ and $c_{0}>0$ such that

$$
c f\left(u_{0}\right) \neq \rho_{K}\left(u_{0}\right)-\rho_{K}\left(-u_{0}\right),
$$

for all $c$ with $0<c \leq c_{0}$; note that this ensures that $\rho_{L}\left(u_{0}\right) \neq \rho_{K}\left( \pm u_{0}\right)$ for the corresponding bodies.

It remains to show that we can also choose $c$ so that $L$ is convex. To see this, note that it suffices to show that $L \cap S$ is convex for each two-dimensional subspace, so we may assume that $n=2$ and use polar coordinates. By the formula for curvature in polar coordinates, we have

$$
2 \rho_{K}^{\prime}(\theta)^{2}-\rho_{K}(\theta) \rho_{K}^{\prime \prime}(\theta)+\rho_{K}(\theta)^{2}>0,
$$

for $0 \leq \theta \leq 2 \pi$. Now for $\theta \in U$, we have $\rho_{L}(\theta)=\rho_{K}(\theta)-c f(\theta)$ and hence

$$
2 \rho_{L}^{\prime}(\theta)^{2}-\rho_{L}(\theta) \rho_{L}^{\prime \prime}(\theta)+\rho_{L}(\theta)^{2}=2 \rho_{K}^{\prime}(\theta)^{2}-\rho_{K}(\theta) \rho_{K}^{\prime \prime}(\theta)+\rho_{K}(\theta)^{2}+c F(\theta),
$$

where $F=F\left(f, f^{\prime}, f^{\prime \prime}, \rho_{K}, \rho_{K}^{\prime}, \rho_{K}^{\prime \prime}\right)$ is a polynomial. Therefore we can choose $c \leq c_{0}$ sufficiently small to ensure that

$$
2 \rho_{L}^{\prime}(\theta)^{2}-\rho_{L}(\theta) \rho_{L}^{\prime \prime}(\theta)+\rho_{L}(\theta)^{2} \geq 0,
$$


for $\theta \in U$. For $\theta \in-U$, we have $\rho_{L}(\theta)=\rho_{K}(\theta)+g_{c}(-\theta)$ and

$$
2 \rho_{L}^{\prime}(\theta)^{2}-\rho_{L}(\theta) \rho_{L}^{\prime \prime}(\theta)+\rho_{L}(\theta)^{2}=2 \rho_{K}^{\prime}(\theta)^{2}-\rho_{K}(\theta) \rho_{K}^{\prime \prime}(\theta)+\rho_{K}(\theta)^{2}+G(\theta),
$$

where $G=G\left(g_{c}, g_{c}^{\prime}, g_{c}^{\prime \prime}, \rho_{K}, \rho_{K}^{\prime}, \rho_{K}^{\prime \prime}\right)$ is a polynomial. It is easy to check that as $c \rightarrow 0$, $g_{c}$ and its first two derivatives converge to zero uniformly in $\theta$ and that this implies that $G$ also converges to zero uniformly in $\theta$. Therefore we can choose $c$ sufficiently small to ensure that also

$$
2 \rho_{L}^{\prime}(\theta)^{2}-\rho_{L}(\theta) \rho_{L}^{\prime \prime}(\theta)+\rho_{L}(\theta)^{2} \geq 0
$$

for $\theta \in-U$, and hence that $L$ is convex.

The class of convex bodies in $\mathcal{K}_{o}^{n}$ with everywhere positive Gaussian curvature is dense in $\mathcal{K}_{o}^{n}$ (this follows from Theorem 3.3.1 of [18]). On the other hand, most members of $\mathcal{K}_{o}^{n}$ have zero Gaussian curvature at almost every point on their boundaries. This is a result of Zamfirescu (see p. 1332 of [12] and the references given there).

Theorem 5.4. Let $T$ be a triangle in $\mathbb{E}^{2}$ with centroid at the origin. Then $T$ is determined in $\mathcal{K}_{o}^{2}$, up to reflection in the origin, by its section function (X-ray at the origin).

Proof. By applying a linear transformation, if necessary, we may assume that $T$ has vertices $(1,0),\left(-\frac{1}{2}, \sqrt{3} / 2\right)$, and $\left(-\frac{1}{2},-\sqrt{3} / 2\right)$. Suppose that $K \in \mathcal{K}_{o}^{2}$ has the same section function as $T$.

We work with the radial function of $K$ in polar coordinates. Let $x_{n}=\rho_{K}(n \pi / 3)$, so that

$$
x_{n+6}=x_{n},
$$

for all $n \in \mathbb{N}$. Since $K$ has the same section function as $T$, we also have

$$
x_{n}+x_{n+3}=\frac{3}{2}
$$

and

$$
0<x_{n}<\frac{3}{2}
$$

for all $n \in \mathbb{N}$. Our goal is to prove that either (i) $x_{1}=\frac{1}{2}, x_{2}=1$, and $x_{3}=\frac{1}{2}$, or (ii) $x_{1}=1, x_{2}=\frac{1}{2}$, and $x_{3}=1$. If (i) holds, then by (7) the vertices of $T$ belong to $K$, so $T \subset K$, and the equality of the section functions then implies $K=T$. If (ii) holds, the vertices of $-T$ belong to $K$, so we conclude similarly that $K=-T$.

We first establish two more relations among the $x_{n}$ 's. The convexity of $K$ yields (see Lemma 5.1 .4 of [5])

$$
\frac{1}{x_{n+1}} \leq \frac{1}{x_{n}}+\frac{1}{x_{n+2}}
$$

and

$$
\frac{\sqrt{3}}{\rho_{K}((2 n+1) \pi / 6)} \leq \frac{1}{x_{n}}+\frac{1}{x_{n+1}},
$$


for all $n \in \mathbb{N}$. Since $K$ has the same section function as $T$, we have

$$
\rho_{K}\left(\frac{(2 n+1) \pi}{6}\right)+\rho_{K}\left(\frac{(2 n+7) \pi}{6}\right)=\frac{2 \sqrt{3}}{3},
$$

so the previous inequality gives

$$
\frac{\sqrt{3}}{1 / x_{n}+1 / x_{n+1}}+\frac{\sqrt{3}}{1 / x_{n+3}+1 / x_{n+4}} \leq \frac{2 \sqrt{3}}{3} .
$$

Combining this with (7) we have

$$
\frac{\sqrt{3}\left(3 x_{n}+3 x_{n+1}+16 x_{n} x_{n+1}-10 x_{n}^{2}-10 x_{n+1}^{2}\right)}{12\left(x_{n}+x_{n+1}\right)\left(3-\left(x_{n}+x_{n+1}\right)\right)} \leq 0,
$$

so, by (8),

$$
3 x_{n}+3 x_{n+1}+16 x_{n} x_{n+1}-10 x_{n}^{2}-10 x_{n+1}^{2} \leq 0
$$

for all $n \in \mathbb{N}$. Note that (10) is satisfied unless $\left(x_{n}, x_{n+1}\right)$ lies in the interior of an ellipse $E$ in $\mathbb{E}^{2}$ that is symmetric with respect to the line $y=x$. The ellipse $E$ contains $(0,0)$, $\left(0, \frac{3}{10}\right),\left(\frac{1}{10}, \frac{1}{2}\right),\left(\frac{1}{2}, 1\right),\left(1, \frac{7}{5}\right)$, and $\left(\frac{3}{2}, \frac{3}{2}\right)$ in its boundary, and therefore also contains the squares $S_{1}=\left\{(x, y): 0 \leq x, y \leq \frac{3}{10}\right\}, S_{2}=\left\{(x, y): \frac{1}{10} \leq x, y \leq \frac{1}{2}\right\}$, and $S_{3}=\left\{(x, y): \frac{1}{2} \leq x, y \leq 1\right\}$.

We prove that either (i) or (ii) holds by a series of claims.

Claim 1. If $\frac{1}{2} \leq x_{n}, x_{n+1} \leq 1$ for any $n \in \mathbb{N}$, then $x_{n}=\frac{1}{2}$ and $x_{n+1}=1$ or vice versa.

This is an immediate consequence of the fact that $S_{3} \subset E$.

Claim 2. It is not possible that $x_{n}, x_{n+1} \leq \frac{1}{2}$ for any $n \in \mathbb{N}$.

Observe first that since $E$ contains the squares $S_{1}$ and $S_{2}$, we have $\frac{3}{10}<x_{n} \leq \frac{1}{2}$ and $x_{n+1}<\frac{1}{10}$ or vice versa. If the former possibility holds, then, by (4),

$$
\frac{1}{x_{n+2}} \geq \frac{1}{x_{n+1}}-\frac{1}{x_{n}}>\frac{1}{\frac{1}{10}}-\frac{1}{\frac{3}{10}}=\frac{20}{3},
$$

so $x_{n+2}<\frac{3}{20}$. Since $x_{n+1}<\frac{1}{10}$, we must have $\frac{3}{10}<x_{n+2} \leq \frac{1}{2}$, by the above argument with $n$ replaced by $(n+1)$, and this is a contradiction. The assumption that $0<x_{n}<\frac{1}{10}$ and $\frac{3}{10}<x_{n+1} \leq \frac{1}{2}$ similarly leads to a contradiction, so Claim 2 is proved.

Claim 3. If $1 \leq x_{n}, x_{n+2}$ and $x_{n+1} \leq \frac{1}{2}$ for any $n \in \mathbb{N}$, then $x_{n}=1, x_{n+1}=\frac{1}{2}$, and $x_{n+2}=1$.

This follows immediately from (9).

Claim 4. If $\frac{1}{2} \leq x_{n} \leq 1, x_{n+1} \leq \frac{1}{2}$, and $1 \leq x_{n+2} \leq \frac{3}{2}$ for any $n \in \mathbb{N}$, then $x_{n}=1$, $x_{n+1}=\frac{1}{2}$, and $x_{n+2}=1$. 
Note that $x_{n} \leq 2 x_{n+1}$, for otherwise, by (9),

$$
\frac{1}{x_{n+1}} \leq \frac{1}{x_{n}}+\frac{1}{x_{n+2}} \leq \frac{1}{x_{n}}+1<\frac{1}{2 x_{n+1}}+1 \leq \frac{1}{2 x_{n+1}}+\frac{1}{2 x_{n+1}}=\frac{1}{x_{n+1}} .
$$

The line $y=x / 2$ meets bd $E$ at the origin and $\left(1, \frac{1}{2}\right)$, so the triangle determined by this line and the lines $x=\frac{1}{2}$ and $y=\frac{1}{2}$ is contained in $E$ and intersects bd $E$ only at $\left(1, \frac{1}{2}\right)$. It follows that if $\frac{1}{2} \leq x_{n} \leq 1, x_{n+1} \leq \frac{1}{2}$, and $x_{n} \leq 2 x_{n+1}$, then (10) holds only if $x_{n}=1$ and $x_{n+1}=\frac{1}{2}$, in which case $x_{n+2}=1$ by (9).

We now represent any sequence $\left\{x_{n}\right\}$ by a sequence of letters $S, M$, and $L$, each letter being used at the $n$th place if $x_{n} \leq \frac{1}{2}, \frac{1}{2} \leq x_{n} \leq 1$, or $1 \leq x_{n}$, respectively. Each sequence is determined by the first three letters; thus if the sequence begins $S, L, M$, it must continue $L, S, M$, by (7), and then repeat by (6). Thus we have 27 different possible sequences. Claims 1-4 above each exclude sequences containing some concatenation of letters. Specifically, the appearance of $M, M$, or $S, S$, or $L, S, L$, or $M, S, L$, is forbidden by Claims 1-4, respectively, with certain exceptional values of $x_{n}$ allowed as in the statements of the claims. Each of the 27 sequences can now be examined individually. For example, the sequence above beginning $S, L, M$ continues with $L, S$, $M, S, L, M$ and therefore contains $M, S, L$. By Claim 4 this is only possible when $x_{6}=1, x_{7}=\frac{1}{2}$, and $x_{8}=1$, so using (6) and (7), we see that (i) holds . A routine check shows that in each case (i) or (ii) holds, completing the proof.

If it is true that a set of convex polytopes dense in $\mathcal{K}_{o}^{n}$ are determined in $\mathcal{K}_{o}^{n}$, up to reflection in the origin, by their section functions, then a standard argument shows that the answer to Question 5.2 is affirmative. In view of Theorem 5.3, however, this may be false or difficult to prove. There are certainly convex polygons whose vertices are in general position with respect to the origin that are not determined in this sense. An example is the pentagon $P$ depicted in Fig. 2.

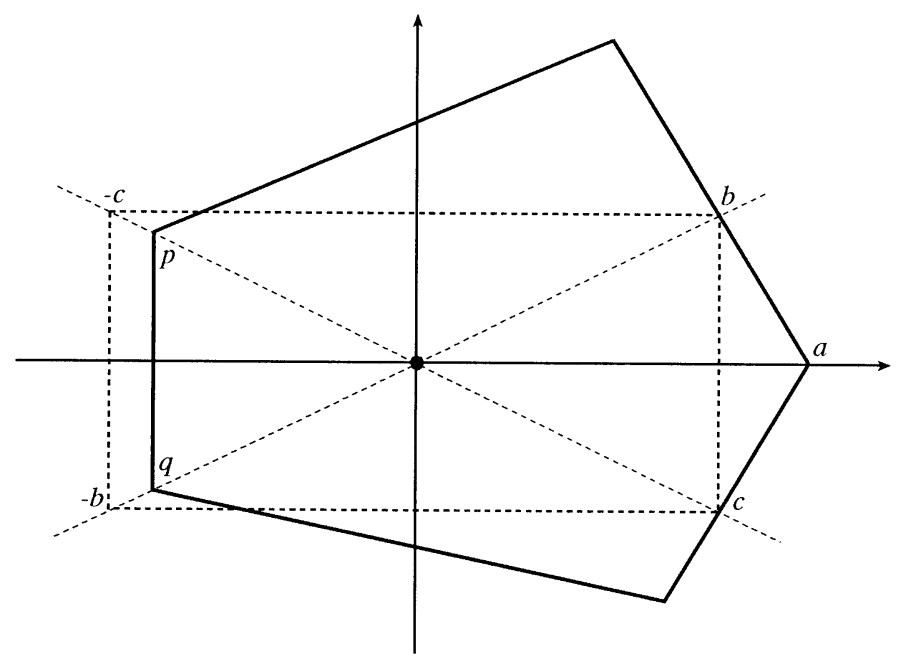

Fig. 2. The pentagon $P$. 
To see that $P$ is not determined in $\mathcal{K}_{o}^{2}$, up to reflection in the origin, by its section function, Minkowski's inequality can be used as in the proof of Theorem 5.1 to show that there is a convex body $H$, whose intersection with $P$ is precisely the segment $[p, q]$, such that $H$ has the same section function as the reflection in the origin of the triangle $T$ with vertices at $a, b$, and $c$. The body $K$ formed by removing $T$ from $P$ and adding $H$ then has the same section function as $P$. Moreover, the interior angles of $P$ at $p$ and $q$ are sufficiently small to ensure that $K$ is convex.

\section{References}

1. C. Bauer, Determination of convex bodies by projection functions, preprint.

2. C. Bauer, Intermediate surface area measures and projection functions of convex bodies, Arch. Math. 64 (1995), 69-74.

3. G. D. Chakerian and E. Lutwak, Bodies with similar projections, Trans. Amer. Math. Soc. 349 (1997), $1811-1820$.

4. R. J. Gardner, X-rays of polygons, Discrete Comput. Geom. 7 (1992), 281-293.

5. R. J. Gardner, Geometric Tomography, Cambridge University Press, New York, 1995.

6. R. J. Gardner, Geometry tomography, Notices Amer. Math. Soc. 42 (1995), 422-429.

7. R. J. Gardner and A. Volčič, Determination of convex bodies by their brightness functions, Mathematika 40 (1993), 161-168.

8. R. J. Gardner and A. Volčič, Tomography of convex and star bodies, Adv. in Math. 108 (1994), 367-399.

9. P. R. Goodey, R. Schneider, and W. Weil, Projection functions of convex bodies, Intuitive Geometry (Budapest, 1995), Bolyai Mathematical Studies 6, Budapest, 1997, pp. 23-53.

10. P. R. Goodey, R. Schneider, and W. Weil, On the determination of convex bodies by projection functions, Bull. London Math. Soc. 29 (1997), 82-88.

11. P. R. Goodey and W. Weil, Intersection bodies and ellipsoids, Mathematika 84 (1995), 295-304.

12. P. M. Gruber, Baire categories in convexity, in Handbook of Convex Geometry, ed. by P. M. Gruber and J. M. Wills, North-Holland, Amsterdam, 1993, pp. 1327-1346.

13. G. H. Hardy, J. E. Littlewood, and G. Pólya, Inequalities, Cambridge University Press, Cambridge, 1959.

14. D. A. Klain, Star valuations and dual mixed volumes, Adv. in Math. 121 (1996), 80-101.

15. K. Kuratowski, Topology, Vol. II, Academic Press, Warsaw, 1968.

16. E. Lutwak, Dual mixed volumes, Pacific J. Math. 58 (1975), 531-538.

17. E. Lutwak, Intersection bodies and dual mixed volumes, Adv. in Math. 71 (1988), 232-261.

18. R. Schneider, Convex Bodies: The Brunn-Minkowski Theory, Cambridge University Press, Cambridge, 1993.

19. R. Schneider, Polytopes and Brunn-Minkowski theory, in Polytopes: Abstract, Convex and Computational, ed. by T. Bisztriczky et al., NATO ASI Series, vol. 440, Kluwer, Dordrecht, 1994, pp. 273-299.

Received December 4, 1996, and in revised form April 14, 1997. 